\title{
Using Energy-Efficient Overlays to Reduce Packet Error Rates in Wireless Ad-Hoc Networks
}

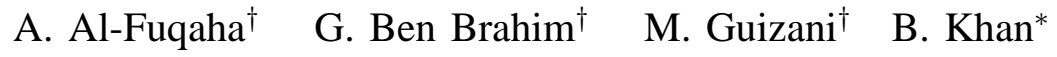

\begin{abstract}
In this paper we present new energy-efficient techniques to lower the packet-level error rates of applicationlayer connections in wireless ad-hoc networks. In our scheme, each application-layer connection is implemented at the physical level by an overlay network. Data packets submitted at the connection source are checksummed and replicated, flowing breadth-first across the overlay network towards the destination. The destination delivers the first error-free copy of each packet, in order, to the application layer, dropping packets that are corrupt or duplicate. Specifically in this paper, we consider overlays consisting of multiple parallel node-disjoint multi-hop paths. We compare this overlay scheme with the traditional scheme in which the source transmits to the destination along a single minimum-hop path. We show that even when the two schemes are constrained by identical power consumption bounds, an overlay scheme that uses multiple multi-hop paths provides significantly lower packet-level error rates in many common situations. We describe the relationship between packet error rate, the number of paths, and the lengths of each path, and show that the qualitative nature of the relationship changes significantly, depending on available power budget.
\end{abstract}

\section{INTRODUCTION}

Power allocation is of vital importance in wireless ad-hoc networks. Ongoing advances in applications of wireless computing/communication devices drive the energy requirements of these systems ever upwards. Clearly the capacity of the batteries which power most mobile devices creates a hard constraint on the operational time of a mobile computing system. Not surprisingly, this makes the design of energy efficient mobile ad-hoc networks an important area of current research.

Lowering energy consumption indiscriminately, however, can have undesirable side effects, most notably, it can raise the bit error (and hence packet-level error) rate of connections. Since many applications require very specific Quality of Services (QoS), such a degradation could yield the network inoperable.

In this paper, we consider the problem of how to balance

$\dagger$ Western Michigan University, MI.

* John Jay College of Criminal Justice, City University of New York, NY 10019 . the need for efficient energy allocation with the objective of low packet-level error rates.

\section{RELATED WORK}

Approaches for efficient power management in wireless networks, have been investigated [4], [16], [18] at the various protocol layers. For example:

(1) Physical layer: Using directional antennae, applying knowledge of spatial neighborhood as a hint in setting transmission power,

(2) Data-link layer: Avoiding unnecessary retransmissions, avoiding collisions in channel access whenever possible, allocating contiguous slots for transmission and reception whenever possible,

(3) Network layer: Considering route-relay load, considering battery life in route selection, reducing frequency of control messages, optimizing size of control headers, route reconfiguration, and

(4) Transport layer: Avoiding repeated retransmissions, handling packet loss in a localized manner, using powerefficient error control schemes.

Topology control and management is one of the techniques used for efficient power usage [8]. This approach consists of determining the transmission power of each node so as to maintain network connectivity while consuming the minimum possible power. Instead of transmitting using the maximum possible power, nodes in a wireless multi-hop network collaboratively determine their transmission power and define the topology of the wireless network by the neighbor relation under certain criteria. This is in contrast to the traditional network in which each node transmits using its maximum transmission power and the topology is built implicitly by routing protocols without considering the power issue.

Another approach for minimizing the power usage in wireless network is to reduce the amount of communication between nodes at the expense of extra computation. Most work focused on developing approaches that reduce the volume of 
data that need to be transmitted, typically through intelligent data reduction and aggregation techniques.

Another category of solutions have been proposed at the network layer, which consists of designing energy aware routing protocols [6], [9], [16]. In wired networks, the emphasis has traditionally been on maximizing end-to-end throughput and minimizing delay. In general, paths are computed based on minimizing hop count or delay. Nonetheless, to maximize the lifetime of mobile hosts, routing algorithms must select the energy efficient paths. Hence, routes requiring lower levels of power transmission are preferred, but this can affect endto-end throughput. Transmission with higher power increases the probability of successful transmission, thus increasing the end-to-end throughput. However, it also yields higher interference with other mobile hosts, which can destroy an existing transmission band and may cause the network to have blocked calls. This could result in a decrease in network capacity. Therefore, lower power transmission does not always have a negative impact on throughput. Since lower power transmission can reduce channel interference and contentions, it can increase end-to-end throughput. When power efficiency is considered, ad hoc networks will require a routing algorithm that can evenly distribute packet-relaying loads to each node to prevent nodes from being overused.

In [15], Tang el al. studied energy efficient algorithms for survivable broadcast/multicast routing, that are resilient to a single node failure. The network survivability was assured by applying redundant trees. The authors proposed the minimax survivable broadcasting/multicasting problems and the minimum survivable broadcasting/multicasting problem.

In [3], [5], Misra and Banerjee argued that energy-aware routing algorithms that are solely based on the energy spent in a single transmission are not able to find minimum energy paths for end-to-end reliable packet transmissions. They considered the case of End-to-End Retransmission and Hopby-Hop Retransmission. They have shown why the effective total transmission energy, which includes the energy spent in potential retransmissions is the proper metric for reliable and energy efficient communication.

In [14], Tang and Xue studied the tradeoff between the path lifetime and the total energy in wireless networks. They proposed two algorithms. The first algorithm constructs a pair of node disjoint paths whose total energy is minimum under the constraint that the lifetime is no smaller than a given threshold. The second algorithm computes a pair of node disjoint paths whose lifetime is maximum under the constraint that the total energy consumption is bounded by a given threshold. Their work was based on Srinivas and Modiano's [13], where they presented an efficient source transmit power selection algorithm, which finds the node disjoint paths with minimal total energy.

In [17], Shibo et al. showed that the performance of a protocol for an ad-hoc network can be enhanced if the protocol is designed based on overlaying a virtual infrastructure on the ad-hoc network. Therefore, in our current work, we propose designing an overlay network on top of a wireless physical network such that the total signal power required to establish several connection requests is minimized while still providing a good QoS in terms of BER.

\section{APPROACH}

Our ultimate goal is to develop energy-efficient routing protocol which guarantees good quality of service in mobile ad-hoc wireless networks. To obtain insight into the logic of routing, this paper begins by considering the dual problem: design. Suppose we wish to establish a connection between two wireless nodes: a source node $s$, and a destination node $t$ that is situated $D$ meters away. If we are given a power "budget" of $P$ Watts for the connection, what is the best way to use this power budget to reduce bit and packetlevel error rates? The answer to this network design question contains important guidelines for routing-when the placement of intermediate nodes cannot be "designed" at will, but rather must be chosen from a predetermined set.

Suppose we use a $k$-hop Overlay Scheme: At the physical level the virtual link from $s$ to $t$ is implemented by a $k$-hop path (i.e., using $k-1$ uniformly spaced intermediate relay nodes) and $1 / k$ of the total power budget $P$ has been assigned to each of these relays (and to $s$ ). With this scheme, which is based on [3], we neglect the power used by the devices to receive the signal, which is assumed to be small compared to the power used during the transmission. Let us denote the bit error rate of such a connection from $s$ to $t$ as $B E R(k)$. Question 1, Given a fixed power budget $P$, for what value of $k$ is $B E R(k)$ minimum?

Suppose we now consider a more general $(N, k)$ Overlay Scheme: At the physical level the virtual link from $s$ to $t$ is implemented by $N$ node-disjoint $k$-hop paths between $s$ and $t$ (each consisting of $k-1$ uniformly spaced relay nodes as shown in Figure 1). Suppose we allocate a $1 /(N(k-1)+1)$ fraction of the total power budget $P$ to each of the relays (and to $s$ ). The network overlay implements a virtual link from $s$ to $t$ as follows: the source $s$ replicates the data packets over all $N$ paths. The destination $t$ delivers the first error-free copy of each packet, in order, dropping any packets that are corrupt or duplicate. Denote the bit error rate of such a connection from 


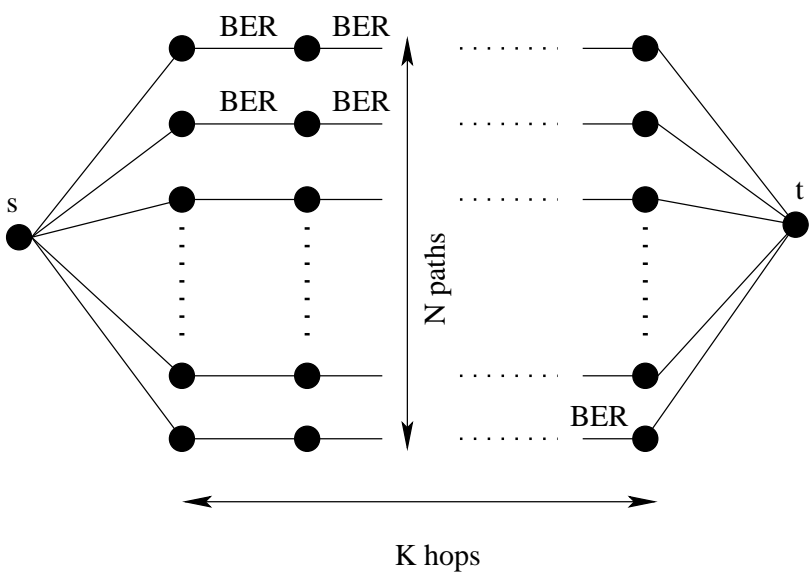

Fig. 1. Virtual link of size $\mathrm{N}$ disjoint paths and k-hops each

$s$ to $t$ as $B E R(N, k)$. Question 2, Given a fixed power budget $P$, for what values of $N$ and $k$ is $B E R(N, k)$ minimum?

We shall see that the answers to these questions depend greatly on the environmental circumstances in which they are asked. To begin, we review some of the mathematical foundations of the problem.

\section{MAThematical Foundations}

The packet error rate over a wireless link is given by

$$
P E R=1-(1-B E R)^{L},
$$

where $L$ is the packet size in bits and $B E R$ is the bit error rate of the channel. For small $B E R$, we can assume a linear approximation, $P E R=L \cdot B E R$. Minimizing $P E R$ is thus attainable by simply minimizing $B E R$; in what follows we restrict our consideration to this objective.

Suppose we have a pair of nodes at distance $D$ communicating using transmitted signal power $P$ over a wireless channel with noise power $P_{\text {noise }}$ and antenna/medium constant* ${ }^{*}$ The relationship between $B E R$ over a wireless channel and the received power $P_{r c v}$ is a function of the modulation scheme. Here we consider a non-coherent binary orthogonal Frequency Shift Keying (FSK) modulation scheme ${ }^{\dagger}$ for which the instantaneous channel bit error rate is [7], [10], [11]:

$$
B E R=\frac{1}{2} e^{-\frac{P_{\text {rcv }}}{2 P_{\text {noise }}}}
$$

where the received power $P_{r c v}=\frac{P}{D^{\alpha}}$.

\footnotetext{
${ }^{*} \alpha$ is typically around 2 for short distances and omnidirectional antennae, and around 4 for longer distances.

$\dagger$ Other modulation schemes can be analyzed in similar way, though closedform analysis may not always be possible.
}

The bit error rate of a virtual link implemented by $N$ disjoint paths of length $k$ is given by

$$
\operatorname{BER}(N, k)=\left(1-(1-B E R)^{k}\right)^{N}
$$

The total power required for transmissions on the virtual link must exceed $(N(k-1)+1)$ times the transmission power at each relay node (and $s$ ). Since each relay must transmit with power at least $P_{r c v}(D / k)^{\alpha}$, but expression (2) indicates that the received power must be at least $-2 P_{\text {noise }} \ln (2 \cdot B E R)$, it follows that the total transmission power $P$ must be at least

$$
-2 P_{\text {noise }} D^{\alpha} \frac{((k-1) N+1)}{k^{\alpha}} \ln (2 \cdot B E R) .
$$

Solving expression (4) for $B E R$ and expressing the result in terms of the channel quality

$$
\gamma=\frac{P}{P_{\text {noise }} D^{\alpha}}
$$

we get that

$$
B E R=\frac{1}{2} e^{-\frac{\gamma}{2} \frac{k^{\alpha}}{((k-1) N+1)}} .
$$

Finally, combining this with expression (3), we get that

$$
\operatorname{BER}(N, k)=\left(1-\left(1-\frac{1}{2} e^{-\frac{\gamma}{2} \frac{k^{\alpha}}{((k-1) N+1)}}\right)^{k}\right)^{N}
$$

\section{NumericAl ANALYSis}

The results in this section are all based on numerical analysis of expression (7), and address Questions 1 and 2 introduced in Section III. Before beginning the numerical analysis, we determine the real-world ranges of our study parameters:

- $\alpha$, the medium/antennae constant, is taken to be 2 .

- $D$, the distance between adjacent hops is taken to be $10-200$ meters, commensurate with technologies such as WaveLAN [2] and Bluetooth device transceivers [1].

- $P_{\min }$, the minimum power receivable by a wireless device at the maximum transmission range, and is taken to be from $-100 \mathrm{dBm}$ to $70 \mathrm{dBm}$ (or $10^{-10}$ to $10^{-7}$ Watts). For example, $P_{\min }$ is $3.65 \cdot 10^{-10}$ Watts for WaveLAN cards [2], and around $-70 \mathrm{dBm}$ for Bluetooth transceivers [1].

- $S N R$, the Signal to Noise Ratio which ranges from 0.11 $\mathrm{dB}$ to $25 \mathrm{dBm}$ for wireless channels.

- $\gamma$, the channel quality, ranges between 0 and 5; these bounds are based on the bounds on $D$ and the $S N R$.

Figure 2 shows how the virtual link BER changes as the number of disjoint paths increases from 1 to 20 for small and large values of $\gamma$. For small $k$, BER decreases monotonically as 
the number of alternate paths increases. For large $k$, however, BER rises until it reaches a local maximum, after which increasing the number of disjoint paths improves the overall BER. Comparing the heights of the curves in the bottom chart, we conclude that when the channel conditions are poor (low values of $\gamma$ ), shorter paths result in better overall BER. By considering the intersections of the curves in top chart, we see that for $k \geq 2$ hops, virtual links with longer path lengths achieve better BER than those with shorter path lengths.
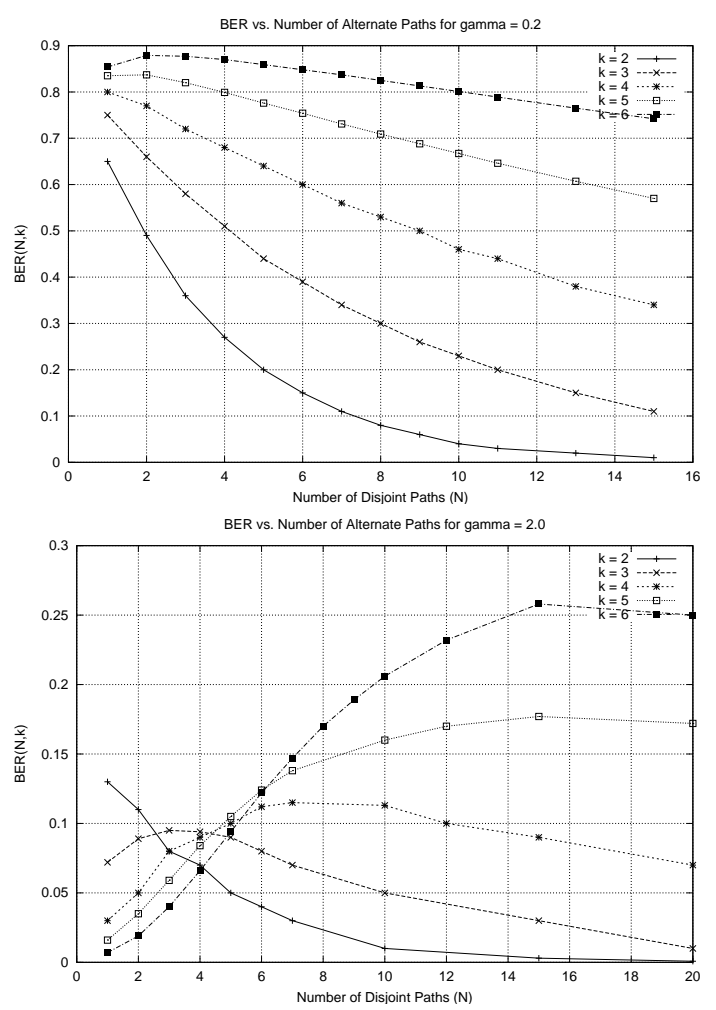

Fig. 2. Virtual link BER vs. number of disjoint paths for various $\gamma$

The analysis of Figure 2 naturally leads one to introduces a concept of a critical density $N_{(k, \gamma)}$ : the minimum number of disjoint paths (having $k$ hops each) that have to be established between nodes $s$ and $t$, so that a better BER is achieved than with a single $k$-hop path with the same power budget. The next graph (figure 3) illustrates critical density as a function of $k$ under various values of $\gamma$.

From Figure 3, we see that (i) for any fixed $\gamma$ the critical density increases exponentially with the number of intermediate hops per path; (ii) for fixed $k$, critical density grows superlinearly with respect to $\gamma$.

Figure 4 shows how the optimal $k$ depends on the values of $\gamma$. Considering the top chart, we conclude that for small $\gamma$, the BER increases as the number of intermediate hops per path increases. Comparing the heights of the curves in the top

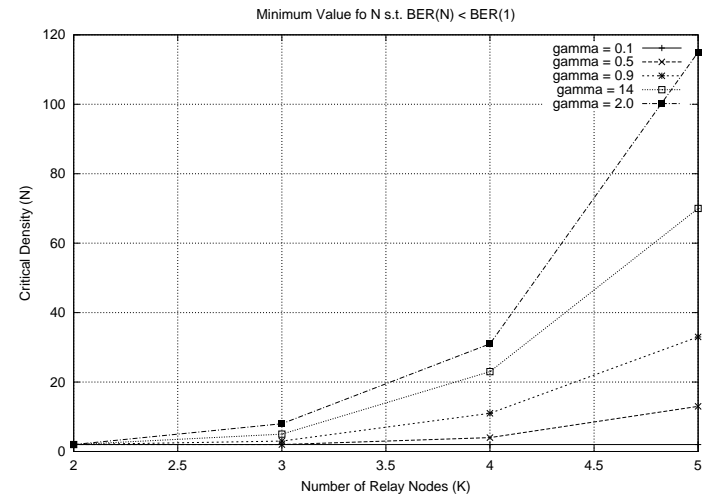

Fig. 3. Variation of the critical density with path length for different $\gamma$.

chart, we conclude that for poor wireless channel conditions, increasing the number of disjoint paths between pairs of source and destination nodes results in better BER.

In case of better wireless channel conditions, however, the bottom chart of figure 4 shows that BER initially increases with $k$ (to a local maximum) and then eventually decreases. When $k$ is small, virtual links with a large number of disjoint paths outperform those with fewer disjoint paths, but this ranking reverses for large $k$. This could be explained with the fact that, for short paths and large budget power, the quality of wireless channels between neighboring nodes is still good and therefore we can tolerate less power to be consumed. This power saving will be used in establishing additional alternate paths, which will compensate for the degradation of the wireless channels and result to a better virtual link BER. However, as the path length increases, the channel quality between neighboring nodes becomes mediocre. This would favor virtual links with fewer number of alternate paths over those with larger number of alternate paths, while maintaining the same power budget.

In figures 5 and 6 we show how the virtual link BER changes as $\gamma$ varies between 0.1 and 2 for different number of disjoint paths. Comparing the heights of all curves in each chart, we conclude that for low values of $\gamma$, better BER is obtained for short paths. However as $\gamma$ increases, longer paths outperform shorter paths. This could be justified with the fact that, dividing the total power budget between all nodes, still, results in low BER wireless channels quality between adjacent nodes, which favors multi-hop transmission over the direct transmission.

The chart in Figure 5 presents a turnover point $\gamma_{t}$ (around 0.5 ), such that, for $\gamma<\gamma_{t}$, shorter paths outperform longer ones. However, for $\gamma>\gamma_{t}$, longer paths achieve better BER. Comparing the turnover points of figures 5 and 6 , we conclude that, as $N$ increases, $\gamma_{t}$ is shifted to the right, indicating that 

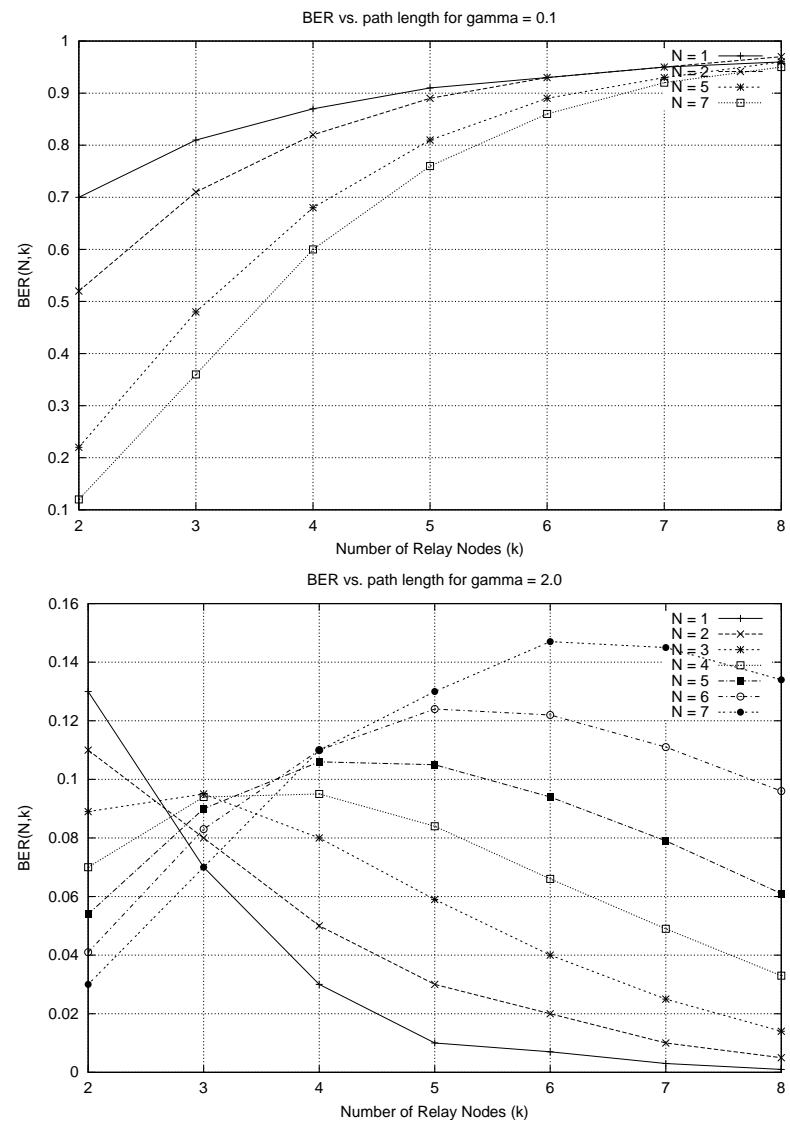

Fig. 4. Virtual BER vs. Path length for various $\gamma$

the range of $\gamma$, where shorter paths outperform longer ones has increased.

\section{CONClusions}

The following three assertions summarize the numerical results we have presented so far.

Assertion 1: If $\gamma \ll 1$, then $\forall k>k^{\prime}$,

$$
\operatorname{BER}(k)>\operatorname{BER}\left(k^{\prime}\right) \text {. }
$$

Assertion 2: If $\gamma \gg 1$, then $\exists k_{\gamma}$ s.t. $\forall k>k^{\prime} \geqslant k_{\gamma}$,

$$
\operatorname{BER}(k)<B E R\left(k^{\prime}\right) \leqslant B E R(1) .
$$

Assertion 3: $\forall k \exists N_{(k, \gamma)}$, such that $\forall N>N^{\prime} \geqslant N_{(k, \gamma)}$

$$
\operatorname{BER}(N, k)<B E R\left(N^{\prime}, k\right) \leqslant B E R(1, k) .
$$

Assertions 1, 2, and 3 are indeed mathematical theorems, and their proofs are immediate from formal asymptotic analysis of expression (7). Assertion 1 (and 2) follow from $(k \rightarrow \infty)$

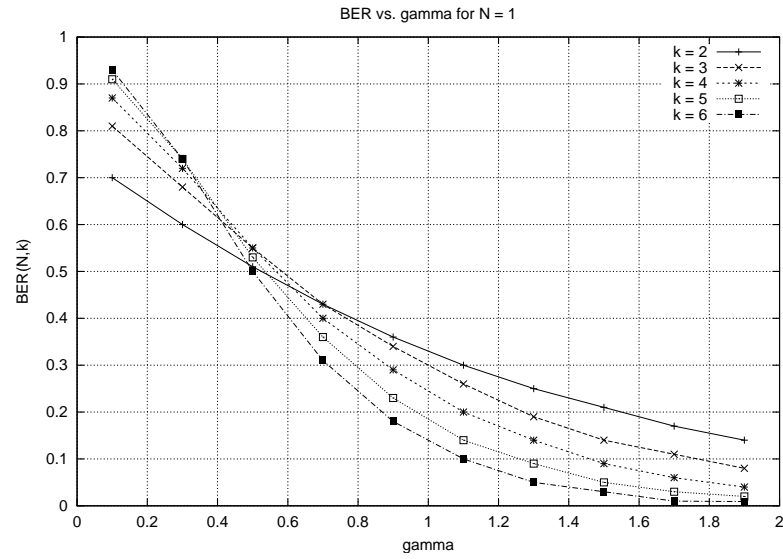

Fig. 5. Virtual Link BER vs. gamma for a single multi-hop path

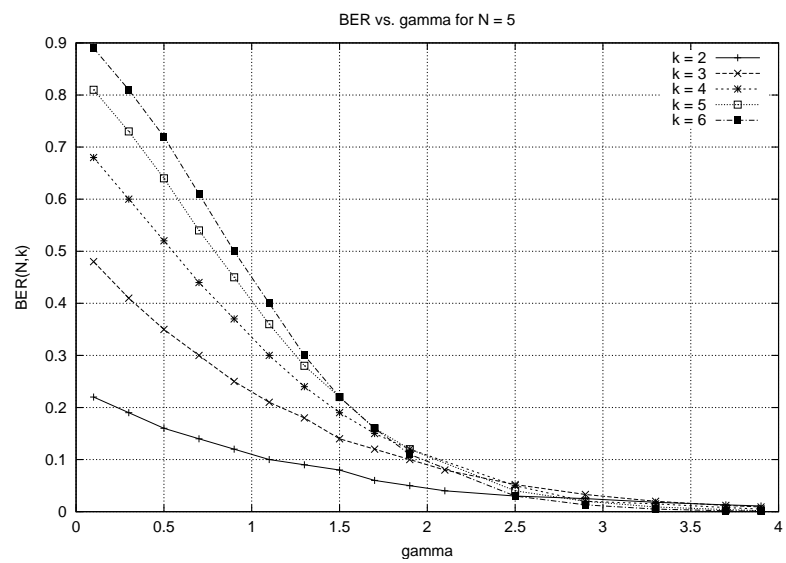

Fig. 6. Virtual Link BER vs. gamma for 5 multi-hop paths

asymptotic analysis when $N=1$ and $\gamma \approx 0$ (resp. $\gamma \geqslant 2$ ). Assertion 3 follows from $(N \rightarrow \infty)$ asymptotic analysis of the same expression for any constant $k$ and $\gamma$. Rather than exposit the formal proofs here, we have chosen to present illustrative numerical studies and interpret the nature of the tradeoffs between the total transmission power and virtual link bit error rate-and explain how these tradeoffs are influenced by the channel quality $\gamma$. Let us now consider their implications.

Assertions 1 and 2 indicate that the optimal value of $k$ depends on a situational parameter we refer to as the channel quality $\gamma$ whose value is proportional to $\frac{P}{D^{\alpha}}$ (where $\alpha \geqslant 2$ is a constant pertaining to transmission medium and antenna design). They give opposing design guidelines depending on the range of $\gamma$ : when $\gamma$ is small, the optimal BER is attained by minimizing $k$, but when $\gamma$ is large, the optimal BER is attained by maximizing $k$.

Consider the implications of Assertion 2 (when $\gamma$ is large). As each relay node transmits with power $P / k$, the downstream 
neighbor $D / k$ meters away receives the transmission with power $\frac{P k^{\alpha-1}}{D^{\alpha}}$. Since a wireless node can receive a transmission only if the power on reception exceeds a threshold minimum power sensitivity $P_{\min }>0$, there exists $k_{\text {min }}$ such that for all $k \geqslant k_{\text {min }}, P_{r c v}(k) \geqslant P_{\text {min }}$. Thus one can achieve arbitrarily low BER while maintaining the same power budget, by simply using ever larger numbers of relay nodes.

Assertion 3 states that, in principle, it is always possible to reduce BER by considering a sufficiently large number of node-disjoint paths. The reader will note that for any fixed $k$ and $\gamma$, if $N \rightarrow \infty$, then $\operatorname{BER}(N, k) \rightarrow 0$. Unfortunately, in practice, it is not possible to achieve an arbitrarily low BER through the establishment of ever larger numbers of parallel node-disjoint paths. To see why, recall that each relay node transmits with signal power $P /(N(k-1)+1)$ Watts, so the downstream neighbor $D / k$ meters away receives the transmission with power

$$
P_{r c v}(N, k)=\frac{P k^{\alpha}-1}{(N(k-1)+1) D^{\alpha}} .
$$

It follows that for $N$ sufficiently large, $P_{r c v}<P_{\text {min }}$, rendering the scheme infeasible: Unfortunately, $P_{\min }>0$ implies that BER cannot be reduced indefinitely while maintaining the same total power budget by using parallel node disjoint paths.

\section{CURRENT TRENDS FOR $\gamma$}

The channel quality $\gamma$ depends on the following parameters: total signal power, the noise power, the distance between the pair of nodes, and the medium/antenna constant. We assume that the signal noise power, distances, antenna/medium constant to be stable in the historical sense. This means $\gamma$ primarily dependent on the total power available per connection. This quantity is clearly expressible as the total power available at each node $P(t)$ divided by the number of connections that the node participates in $\delta(t)$. Unfortunately, projections on progress in battery technology indicate $P(t)$ is expected to grow only modestly in the near future [12]. The number of connections that each node participate in, however, is expected to grow rapidly as new wireless applications are developed and wireless devices gain widespread acceptance. Thus, we anticipate the ratio $P(t) / \delta(t)$, and hence $\gamma$ to decrease over time. As we have seen in the previous section, when $\gamma$ is small, using multiple disjoint paths reduces the BER.

\section{CONCLUSION}

In power-limited wireless ad-hoc networks, battery power is an important consideration to take into account while establishing connections. In this paper, we proposed new energy-efficient techniques to lower the packet-level error rates of application layer connections. Our scheme consists of designing an overlay network on top of the physical network. We consider the case of traditional single multi-hop path overlay and the $(N, k)$ overlay scheme, where at the physical level, the virtual link between two nodes is implemented by $N$ node disjoint k-hop paths. Providing multiple paths for data transfer has an advantage of improving the packet error rate. In our design, the number of disjoint paths between pair of nodes as well as its size is chosen based on a user predefined power consumption bounds. Our numerical analysis show that, for the traditional overlay scheme, minimizing the number of intermediate hops results in better BER when operating in poor wireless channel settings. When wireless channel conditions ameliorate, however, better BER is achieved by maximizing the number of intermediate hops. Another interesting result is that, in several scenarios, the proposed $(N, k)$ overlay scheme outperforms the single muti-hop path scheme under the same total energy budget constraint.

Energy efficient routing has always been a central research topic in wireless network. Most of the prior work has focused on developing routing schemes with the objective of minimizing the total transmission power. We propose using the current results describing the tradeoffs between most parameters involved in the wireless system in the next step of this project, which consists of designing energy-efficient QoSbased routing protocols in mobile ad-hoc wireless network, while minimizing the overall connection BER.

\section{ACKNOWLEDGMENTS}

We would like to acknowledge the US Naval Research Laboratory, Center for Computational Science (Code 5590), Washington DC, for partially funding this research work.

\section{REFERENCES}

[1] Bluetooth resource center. http://www.palowireless.com/infotooth/.

[2] Wavelan/pcmcia card user's guide. Lucent Technologies.

[3] S. Banerjee and A. Misra. Energy Efficient Reliable Communication for Multi-hop Wireless Networks. Journal of Wireless Networks (WINET), 2004.

[4] R. Cravets and P. Krishnan. Power Management Techniques for Mobile Communication. NOBICOM 98 Dallas Texas USA, 1998.

[5] Q. Dong and S. Banerjee. Minimum Energy Reliable Paths Using Unreliable Wireless Links. MobiHoc'05, Urbana-Champaign, Illinois, May 25-27, 2005.

[6] C. E. Jones, K. M. Sivalingam, P. Agrawal, and J. C. Chen. A Survey of Energy Efficient Network Protocols for Wireless Networks. Wireless Networks 7, 343 358, 2001.

[7] G. Laurer. Packet Radio routing, Chapter 11, pages 351-396, Prentice Hall 1995.

[8] N. Li, J. C. Hou, and L. Sha. Design and Analysis of an MST-Based Topology Control Algorithm. IEEE INFOCOM, 2003. 
[9] Q. Li, J. Aslam, and D. Rus. Online Power-aware Routing in Wireless Ad-hoc Networks. Proceedings of ACM Mobicom'2001, pp97-107, 2001.

[10] S. Loyka and F. Gagnon. Performance Analysis of the V-BLAST Algorithm: An Analytical Approach. IEEE Transactions on Wireless Communications, Vol.3 No.4, 2004.

[11] J. G. Proakis. Digital Communications, McGraw Hill, 2001.

[12] S. Sesay, Z. Yang, and J. He. A Survey on Mobile Ad Hoc Wireless Network. Information Technology Journal 3 (2): 168-175, 2004.

[13] A. Srinivas and E. Modiano. Minimum Energy Disjoint Path Routing in Wireless Ad-hoc Networks. MobiCom'03, San Diego, California, September 14-19, 2003.

[14] J. Tang and G. Xue. Node-Disjoint Path Routing in Wireless Networks: Tradeoff between Path Lifetime and Total Energy. IEEE Communications Society, 2004.

[15] J. Tang, G. Xue, and W. Zhang. Energy Efficient Survivable Broadcasting and Multicasting in Wireless Ad hoc Networks. MilCom'04, 2004.

[16] C.-K. Toh. Maximum Battery Life Routing to Support Ubiquitous Mobile Computing in Wireless Ad Hoc Networks. IEEE Communications Magazine, June 2001.

[17] S. Wu and K. S. Candan. GPER: Geographic Power Efficient Routing in Sensor Networks. Proceedings of the 12th IEEE International Conference on Network Protocols (ICNP'04), 2004.

[18] Y. Zhang and L. Cheng. Cross-Layer Optimization for Sensor Networks. New York Metro Area Networking Workshop, New York, September 12, 2003. 